Gefässchirurgie 2011 · 16:9-10

DOI 10.1007/s00772-010-0872-0

Online publiziert: 21. Januar 2011

(c) Springer-Verlag 2011

\section{E.S. Debus}

Klinik und Poliklinik für Gefäßmedizin, Universitäres Herzzentrum, Universitätsklinikum Hamburg-Eppendorf, Hamburg

\title{
Vaskuläre Begutachtung
}

Die mit großem Abstand häufigsten vaskulären Erkrankungen sind dem Venensystem zuzurechnen. Dies konnte in mehreren epidemiologischen Untersuchungen gezeigt werden. So wissen wir heute, dass die primäre Varikose den Charakter einer Volkskrankheit hat, mit einer Prävalenz von $10-40 \%$ für Männer und $25-41 \%$ für Frauen. Für die primäre Varikose besteht eine familiäre Häufung, eine genetische Komponente in der Vererbung dieser Erkrankung ist nachgewiesen. In der Bonner Venenstudie an $>3000$ Probanden wiesen 59\% aller Männer und Frauen retikuläre Varizen oder Teleangiektasien auf - allerdings wiesen lediglich 31\% Varizen mit Krankheitswert auf. Bei 3\% bestanden Hautveränderungen, bei $0,7 \%$ ein aktives oder abgeheiltes Unterschenkelgeschwür. Somit hatte immerhin jeder 6. Mann und jede 5. Frau im Alter zwischen 18 und 79 Jahren Symptome einer CVI (C3-C6 nach CEAP).

Die Prävalenz der CVI dagegen unterscheidet sich geschlechtsspezifisch wesentlich deutlicher und variiert in der Literatur je nach untersuchter Population zwischen $<1 \%-17 \%$ für Männer und $<1 \%$ $40 \%$ für Frauen. Studien zur Prävalenz des abgeheilten oder floriden Ulcus cruris belegen eine Prävalenz von $<1 \%-4 \%$ in der Erwachsenenbevölkerung.

In Anbetracht dieser Zahlen ist es nicht verwunderlich, dass die Zahl der Behandlungen in Deutschland hoch sein muss. Da wir keine verlässlichen Daten zu ambulanten Behandlungen in Deutschland haben, müssen wir auf die zahlenmäßig weit niedrigeren stationären Behandlungen zurückgreifen, die aufgrund ihres wahrscheinlich höheren Schweregrades (nach CEAP) vermutlich auch ein verzerrtes Bild über die Behandlungsart abgeben.
So wurden im Jahr 2007 ca. 120.00o Patienten mit einer Varikose oder einer CVI stationär behandelt ( $\bullet$ Tab. 1), dies entspricht einer Krankenhausinzidenz von 145 Behandlungsfällen/100.0oo/Jahr.

\section{> Die Inzidenz von \\ Phlebothrombosen in Deutschland liegt bei ca. 80.000/Jahr und für Lungenembolien bei ca. 40.000/Jahr}

Auch die Inzidenz tiefer Bein-/Beckenvenenthrombosen ist hoch und beträgt in der Erwachsenenbevölkerung ca. 100/100.000/Jahr. Bei etwa $1 / 3$ dieser Thrombosen tritt eine Lungenembolie (LE) auf. in Autopsiestudien beträgt die Inzidenz der LE sogar $>50 \%$. Wichtige erworbene bzw. begünstigende Risikofaktoren für das Auftreten einer Phlebothrombose sind bekannt (u. a. abgelaufene Thrombose, Tumorleiden, Fettleibigkeit, Hormonsubstitution, Schwangerschaft, Immobilisation, operativer Eingriff, Trauma, möglicherweise Varikose). Hinzu kommen angeborene Risikofaktoren wie z. B. Faktor-V-Leiden, ATIII- und
Protein-C-Mangel. Aufgrund der oben genannten Daten muss für Deutschland mindestens von 80.000 Phlebothrombosen/Jahr und etwa 40.0oo Lungenembolien/Jahr ausgegangen werden.

Vor dem Hintergrund dieser epidemiologischen Daten gewinnt die Diskussion um Behandlungsmodalitäten und in diesem Zusammenhang auch die Frage nach Komplikationen im Rahmen der Therapie besondere Bedeutung. Die invasiven Behandlungsoptionen der Varikose sind durch endoluminale Verödungstherapien und rekanalisierende Verfahren nach thrombotischen Verschlüssen erweitert worden, und die Diskussion darum belebt die Fachliteratur. So stellt sich die Frage, ob die Lebensqualität eines $\mathrm{Pa}$ tienten mit PTS und Varikose -auch bei hoher Komorbidität - mit minimal-invasiven Maßnahmen unter Inkaufnahme eines vertretbaren Behandlungsrisikos nicht optimierbar ist.

Allein durch die Anzahl der Behandlungen stellt die rechtliche Auseinandersetzung mit deren Komplikationen ein relevantes, aktuelles Diskussionsthema dar. Unternimmt man eine Literaturrecherche unter dem Stichwort „Begutachtung“,
Tab. 1 Anzahl der aus der Hauptdiagnosengruppe „Varizen der unteren Extremitäten“ (183.-), mit einem „postthrombotischen Syndrom“ (187.0) oder einer „venösen Insuffizienz" (187.2) kodierten Krankenhauspatienten in Deutschland im Jahr 2007

\begin{tabular}{|c|c|c|c|c|}
\hline Freitext der Hauptdiagnosen & ICD-10 & m & w & $\begin{array}{l}\text { Stationäre Behand- } \\
\text { lungsfälle/100.000 Ein- } \\
\text { wohner/Jahr }\end{array}$ \\
\hline $\begin{array}{l}\text { Varizen untere Extremitäten mit Ulzera- } \\
\text { tion und/oder Entzündung }\end{array}$ & $183.0-2$ & 10.073 & 17.487 & 33,6 \\
\hline $\begin{array}{l}\text { Varizen untere Extremitäten ohne Ulzera- } \\
\text { tion oder Entzündung }\end{array}$ & 183.9 & 23.490 & 54.145 & 94,7 \\
\hline Postthrombotisches Syndrom & 187.0 & 441 & 586 & 1,3 \\
\hline Venöse Insuffizienz (chronisch) (peripher) & 187.2 & 986 & 1.635 & 3,2 \\
\hline Varikose und venöse Insuffizienz & & 34.990 & 73.853 & 132,7 \\
\hline
\end{tabular}


so finden sich unter pubmed von 2000 bis 2010 lediglich 25 Nennungen ohne phlebologischen Bezug. Unter medpilot finden sich zwar 756 Artikel und 150 Monografien, aber lediglich 4 Titel beziehen sich auf phlebologische Themen (Daten aus Nüllen und Noppeney in diesem Heft) - eine unverständliche Diskrepanz mit Blick auf die Wichtigkeit dieses Themas!

Es gibt also Anlass genug, um diesen Problemkreis in den Fokus zu stellen. Nahezu jeder von uns wird im Laufe seines Berufslebens mit gutachterlichen Fragen $\mathrm{zu}$ dem hier angesprochenen Thema befasst sein -als Betroffener und als Gutachter. In beiden Szenarien ist eine Detailkenntnis zu dem aktuellen Stand der gutachterlichen Herangehensweise hilfreich. Ich freue mich, dass wir mit Dr. Nüllen und Dr. Noppeney die wichtigsten deutschsprachigen Autoren zu diesem Thema für eine umfassende Übersichtsdarstellung gewinnen konnten. Die Lektüre ist sehr aufschlussreich und daher sehr empfehlenswert.

In diesem Sinne grüße ich Sie herzlich zum neuen Jahr und wünsche Ihnen auch in 2011 viel Erfolg, Glück und Gesundheit!

Ihr

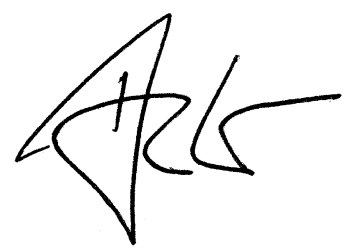

E.S. Debus

Schriftleiter

\section{Korrespondenzadresse}

\section{Prof. Dr. E.S. Debus}

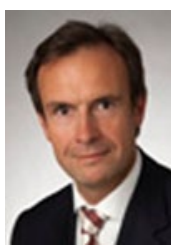

Klinik und Poliklinik für

Gefäßmedizin,

Universitäres Herzzentrum,

Universitätsklinikum

Hamburg-Eppendorf

Martinistr. 52, 20246 Hamburg

debus@uke.de

\section{Springer Award Gefäßmedizin 2011}

\section{Jetzt einreichen! Brückenschlag von experimentellen Ergebnissen zum klinischen Nutzen wird ausgezeichnet}

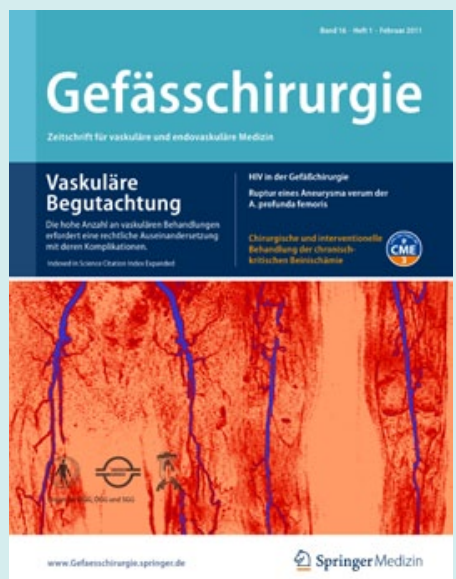

Die Gefäßchirurgie ist ein Wachstumsfach mit zunehmender Bedeutung im Gesamtkonzept der Diagnostik und Therapie von Gefäßerkrankungen. Die wissenschaftliche Leitung der Gefässchirurgie aus Mitgliedern der deutschen, österreichischen und schweizerischen Gesellschaften für Gefäßchirurgie möchten daher zusammen mit dem Springer-Verlag die wissenschaftliche Forschung auf diesem Gebiet unterstützen und insbesondere den Nachwuchs durch die Verleihung des 2. Springer Award Gefäßmedizin fördern.

Nutzen Sie die Gelegenheit und reichen Sie Ihren Beitrag bis zum 01.06.2011 ein. Auf den Preisträger wartet ein Reisestipendium im Wert von 2500,00 EUR!

\section{Teilnahmebedingungen}

- Der Autor soll einen Übersichtsbeitrag in deutscher Sprache auf Grundlage einer eigenen experimentellen Arbeit verfassen. Bei der Beurteilung wird besonderer Wert auf den edukativen Charakter des Artikels sowie die Herausarbeitung der klinischen Relevanz gelegt - der Beitrag soll eine Brücke von den experimentellen Ergebnissen zur klinischen Anwendung schlagen.

- Es können eigene Originaldaten publiziert werden oder auch bereits publizierte eigene Originaldaten zusammengefasst und mit einem Ausblick zur klinischen Relevanz versehen werden. In letzterem Fall ist beim erstpublizierenden Verlag vor Manuskripteinreichung eine Genehmigung einzuholen.

- Die Arbeit wird auf der Jahrestagung der DGG in einer speziellen Sitzung vorgestellt.

- Das Teilnehmeralter ist auf 40 Jahre beschränkt.

- Der Beitrag darf eine Länge von max. 25.000 Zeichen (inkl. Leerzeichen) nicht überschreiten.

Alle eingereichten Beiträge werden durch eine Jury aus dem Editorial Board und dem Herausgebergremium unter der Leitung der Schriftleitung begutachtet.

\section{Preisverleihung im Rahmen der 27. Jahrestagung der DGG}

Die Verleihung des zweiten Springer Award Gefäßmedizin erfolgt im Rahmen der 27. Jahrestagung der Deutschen Gesellschaft für Gefäßchirurgie und Gefäßmedizin vom 14.-17. September 2011 in Erlangen. Vier der besten Arbeiten inklusive der Preisträger-Arbeit werden im Anschluss an die Jahrestagung in Ausgabe 6/2011 der Zeitschrift Gefässchirurgie veröffentlicht.

Bitte reichen Sie Ihren Beitrag zusammen mit Ihrem Lebenslauf in elektronischer Form bei der Verlagsredaktion ein: Tina.Suhai@springer.com

Springer Medizin 